\title{
RULED FUNCTION FIELDS
}

\author{
JAMES K. DEVENEY
}

\begin{abstract}
Let $L=L_{1}\left(x_{1}\right)=L_{2}\left(x_{2}\right) \supset K$ where $x_{i}$ is transcendental over $L_{i}$, and $L_{i}$ is a finitely generated transcendence degree 1 extension of $K, i=$ 1,2. If the genus of $L_{1} / K=0$, then $L_{1}$ and $L_{2}$ are $K$-isomorphic. If the genus of $L_{1} / K>0$, then $L_{1}=L_{2}$ and moreover $L_{1}$ is invariant under all automorphisms of $L / K$. A criterion is also established for a subfield of a ruled field $L$ to be ruled.
\end{abstract}

Let $L$ be a finitely generated extension of a field $K . L$ is said to be ruled over $K$ if there exists an intermediate field $L_{1}$ and an element $x_{1}$ transcendental over $L_{1}$ such that $L=L_{1}\left(x_{1}\right)$. The Zariski problem [6] asks: If $L=L_{1}\left(x_{1}\right)=L_{2}\left(x_{2}\right)$ is ruled in two ways over $K$, must $L_{1}$ and $L_{2}$ be $K$-isomorphic? The answers to some special cases of the 1-dimensional problem were announced in [6] and here we provide a complete affirmative answer for the 1-dimensional case. Henceforth, we assume the transcendence degree of $L$ over $K$ is 2 . If $L_{1}$ is an intermediate field of $L / K$ of transcendence degree 1 over $K$, the genus of $L_{1} / K$ is by definition the genus of $L_{1}$ over the algebraic closure of $K$ in $L_{1}$.

The proof of the one dimensional case is achieved by examining the possibilities for $L$ to be ruled over two distinct subfields $L_{1}$ and $L_{2}$. If $L_{1} \cap L_{2}=K$, then $L_{1}$ and $L_{2}$ must be of genus 0 . This leads to the result that if $L=L_{1}\left(x_{1}\right) \supset L_{1} \supset K$ with $x_{1}$ transcendental over $L_{1}$ and the genus of $L_{1} / K$ is positive, then $L_{1}$ must be invariant under all automorphisms of $L / K$. This result is then used to establish sufficient conditions for a subfield of a ruled field to be ruled ( $K$ not necessarily algebraically closed). Recall, $L$ is regular over $K$ means $L$ is separable over $K$, and $K$ is algebraically closed in $L$.

PROPOSITION 1. Suppose $L=L_{1}\left(x_{1}\right)=L_{2}\left(x_{2}\right) \supset K$ where $x_{i}$ is transcendental over $L_{i}$, and $L_{i}$ is a finitely generated transcendence degree 1 extension of $K, i=1,2$. If $L_{1} \cap L_{2}=K$, then $L_{1}$ and $L_{2}$ are $K$-isomorphic genus 0 extensions of $K$.

ProOF. Since each $L_{i}$ is algebraically closed in $L$, the algebraic closure of $K$ in $L$ is contained in each $L_{i}$. Thus $K$ is algebraically closed in $L$ since $L_{1} \cap L_{2}=K$. By [4, Theorem 1.1, p. 1304], there exists a unique minimal intermediate field $L^{*}$ over which $L$ is separable. Since $L$ is separable over $L_{1}$ and $L_{2}, L^{*} \subseteq L_{1} \cap L_{2}$. Thus $L^{*}=K$, i.e., $L$ is separable over $K$. Thus each $L_{i}$ is separable, hence regular, over $K$. Since $L_{1} \cap L_{2}=K$, we have $L_{1} \not \subset L_{2}$; and therefore some element of $L_{1}$ is transcendental over $L_{2}$. Since the transcendence degree of $L_{1} / K$ is 1 , a transcendence basis for $L_{1} / K$ remains independent over $L_{2}$, i.e., $L_{1}$ and $L_{2}$ are free over $K$. By [5, Theorem 3, p. 57], $L_{1}$ and $L_{2}$ are linearly disjoint over $K$. Now, $L_{2}\left(x_{2}\right) \supseteq L_{2} L_{1} \supset L_{2}$, and hence by Luroth's theorem, $L_{2} L_{1}$ is simple transcendental

Received by the editors October 15, 1981 and, in revised form, January 29, 1982.

1980 Mathematics Subject Classification. Primary 14J25; Secondary 12F20.

Key words and phrases. Ruled field, genus.

(c) 1982 American Mathematical Society 0002-9939/82/0000-0181/801.75 
over $L_{2}$. Thus $L_{2} L_{1}$ is of genus 0 over $L_{2}$. By [3, Theorem 2, p. 132], $L_{1} / K$ is of genus 0 . By a symmetric argument, $L_{2} / K$ is also of genus 0 .

Recall that a genus 0 extension $L_{1}$ of a finite field $K$ is pure transcendental. One sees this as follows: since $L_{1}$ has a divisor of degree 1 [3, Theorem, p. 148] and since the genus is 0 , the corollaries to the Riemann-Roch theorem [3, p. 40] show this divisor must be integral, hence a prime divisor of degree 1 , and hence $L_{1} / K$ is simple transcendental [3, Theorem, p. 50]. Thus if $K$ is finite, $L_{1}$ and $L_{2}$ are simple transcendental extensions of $K$, and hence are isomorphic.

If $K$ is infinite, [7, Lemma 1, p. 209] shows $L_{1}$ is $K$-isomorphic to a subfield of $L_{2}$, and $L_{2}$ is $K$-isomorphic to a subfield of $L_{1}$. If $L_{1}$ is simple transcendental over $K$, then so is $L_{2}$ by Luroth's theorem. If $L_{1}$ is not simple transcendental over $K$, then [1, Corollary 11.3, p. 42] shows $L_{1}$ and $L_{2}$ are $K$-isomorphic. Q.E.D.

It should be noted that if $L_{1}$ is a nonrational genus 0 function field over $K(\operatorname{char} K \neq 2)$ and $L_{2}$ is a $K$-isomorphic copy of $L_{1}$, then $L_{2} L_{1}$, the free join of $L_{1}$ and $L_{2}$, will be ruled over both $L_{1}$ and $L_{2}$ [1, p. 41].

THEOREM 2. Suppose $L=L_{1}\left(x_{1}\right)=L_{2}\left(x_{2}\right) \supset K$ where $x_{i}$ is transcendental over $L_{i}$, and $L_{i}$ is a finitely generated transcendence degree 1 extension of $K, i=1,2$. Then $L_{1}$ and $L_{2}$ are $K$-isomorphic.

PROOF. It suffices to show they are isomorphic over their intersection, which contains $K$. If their intersection is algebraic over $K$, then Proposition 1 applies. If it is not algebraic over $K$, then each of $L_{1}$ and $L_{2}$ must be the algebraic closure in $L$ of their intersection. Thus they are equal in this case.

THEOREM 3. Suppose $L=L_{1}\left(x_{1}\right) \supset L_{1} \supset K$ where $x_{1}$ is transcendental over $L_{1}$ and $L_{1}$ is a finitely generated transcendence degree 1 extension of $K$. Assume the genus of $L_{1} / K>0$. Then $L_{1}$ is invariant under any $K$-automorphism of $L$.

ProOF. Let $\alpha$ be a $K$-automorphism of $L$. Then $L=L_{1}\left(x_{1}\right)=L_{1}^{\alpha}\left(x_{1}^{\alpha}\right)$. Since $L_{1} / K$ is not of genus 0 , Proposition 1 shows $L_{1} \cap L_{1}^{\alpha}$ cannot be algebraic over $K$. But then $L_{1}$ and $L_{1}^{\alpha}$ are both the algebraic closure of $L_{1} \cap L_{1}^{\alpha}$ in $L$, i.e., $L_{1}=$ $L_{1}^{\alpha}$. Q.E.D.

If $L$ is ruled over $K$, must an intermediate field $F$ with $[L: F]<\infty$ also be ruled over $K$ ? If $K$ is algebraically closed of char 0 , [2, Proposition 2, p. 106] shows the answer is yes. For $K$ not algebraically closed (but still of char 0 ), the answer is no. An example is given in [8, p. 330]. There, $K=C(\mu), L=$ $C(\mu, v, w)$ where $\{\mu, v, w\}$ is algebraically independent over $C$. A subfield $F$ with $[C(\mu, v, w): F]=2$ is constructed with $F$ not ruled over $C(\mu)$. Actually, [2] shows $F$ is not pure transcendental over $C(\mu)$. However, if $F$ were ruled, then $F$ would be pure transcendental by the generalized Luroth theorem [6]. However, we can use the results of this paper to get an affirmative answer in some cases.

THEOREM 4. Let $L=L_{1}\left(x_{1}\right) \supset L_{1} \supset K$ where $L_{1}$ is a finitely generated extension of $K$ of transcendence degree 1 and positive genus with $x_{1}$ transcendental over $L_{1}$. Let $G$ be a finite group of $K$-automorphisms of $L$ and let $F$ be its fixed field. If $|G|$ is odd, then $F$ is also ruled over $K$.

Proof. Since $L_{1}$ is invariant under the action of $G$ by Theorem 3, it follows from [8, Theorem 4, p. 322] that $F$ is pure transcendental over $F \cap L_{1}$. 


\section{REFERENCES}

1. S. Amitsur, Generic splitting fields of central simple algebras, Ann. of Math. (2) 62 (1955), 8-43.

2. S. Arima, Double ruled surfaces and their canonical systems, J. Math. Soc. Japan 22 (1970), 105-112.

3. M. Deuring, Lectures on the theory of algebraic functions of one variable, Lecture Notes in Math., vol. 314, Springer-Verlag, Berlin and New York, 1973.

4. J. Deveney and J. Mordeson, Subfields and invariants of inseparable field extensions, Canad. J. Math. 29 (1977), 1304-1311.

5. S. Lang, Introduction to algebraic geometry, Interscience Tracts in Pure and Appl. Math., vol. 5, Interscience, New York, 1958.

6. M. Nagata, $A$ theorem on valuation rings and its applications, Nagoya Math. J. 29 (1967), 85-91.

7. P. Roquette, Isomorphisms of generic splitting fields of simple algebras, J. Reine Angew. Math. 214-215 (1964), 207-226.

8. D. Triantaphyllou, Invariants of finite groups acting non-linearly on rational function fields, $\mathrm{J}$. Pure Appl. Algebra 18 (1980), 315-331.

DEPARTMENT OF Mathematical SCIENCES, Virginia CommonWEalth UNiverSITY, RICHMOND, VIRGINIA 23284 